\title{
Study on vibration and noise influence for optimization of garden mower
}

https://doi.org/10.1515/nleng-2021-0034

Received Jun 10, 2021; accepted Aug 28, 2021.

\begin{abstract}
Advancement in engineering provides various improvement in quality life while taking consideration of important factors for safety and environment. The use of mower food maintenance of land it is very common across several parts of the world with some frequent noise generated through its operation. This article is an attempt to study the noise and frequency generated through the vibrations of mower blade. In this study, an integrated design for designing, testing and developing mower blade that generates less noise is presented. For designing efficient blade that produces less noise, we have implemented various engineering approaches such as rapid product design, process of re-engineering and reverse engineering. The simulation of the designed blade is carried out through CAD software where the design prototype is analysed for its performance. The outcomes of the prototype are tested through simulation and its performance is compared for the determination of success of proposed design at different variations in frequency level. It is observed through the experimentation that the noise and vibration differences are generated through load carrying vehicles, mowers with riding capacity and simple mowers. From the analysis, mower with riding capacity is observed as safest among all other types of machines.
\end{abstract}

Keywords: Computer aided design (CAD), reverse engineering, mower, vibration measurement, noise measurement

\footnotetext{
Yimin Chen, Hebei College of Industry and Technology, Shijiazhuang 050031, China, E-mail: chen.yimin@yahoo.com Wenzhuo Zhang, Hebei College of Industry and Technology, Shijiazhuang 050031, China, E-mail: wenzhuo_z@yahoo.com Lu Dong, Hebei College of Industry and Technology, Shijiazhuang 050031, China, E-mail: donglu29@yahoo.com Korhan Cengiz, Department of Electrical - Electronics Engineering, Trakya University, 22030, Edirne, Turkey, E-mail: korhancengiz@trakya.edu.tr

*Corresponding Author: Amit Sharma, Department of Computer Science and Engineering, Chitkara University, Punjab, India, E-mail: amit.amitsharma90@gmail.com
}

\section{Introduction}

Public gardens maintenance is most important requirement in today's life. In open areas, shrubs are the most common thing and there exist various type of grass cutting machines. The various machines for grass cutting are easily available in the market, such as trimmers, strimmers and grass mowers [1]. These machines cause vibrations and it consists of other risk factors, which may contribute as a risk to the health of workers. In industrial domain, the mechanical vibrations like manufacturing, maintenance and engineering construction maybe termed as an occupational disease for the workers, which are associated with the organisation [2]. In order to overcome vibrations which are associated with the garden mower or other maintenance machines it is essential to understand the state of art behind vibration machines.

To know the activities is relevant to the maintenance of garden, the most common utilised machine are trimmers and mowers [3]. These machines are mostly hand held and therefore accounts hand arm vibration in maximum cases. During study, 250 male workers observed and their health is analysed through questionnaire. Among 250 workers, $95 \%$ of workers observed with sensory neural and vascular disorders [4]. Many of the workers also suffering from issues such as less handgrip strength, finger blanching and numbness. It is also observed from the study that among all of other workers for garden maintenance, the grass cutting workers suffering the most because of vibrations. In one study, the authors have analysed the exposure time of whole body and hand arm vibration at different environments for more than 470 vibration tool workers [5]. This study was completely based on a survey through questionnaire where the daily workers are interviewed and the workplace is observed directly. Among all of the essential tools of gardening, mower has considered as having largest factor of median for the measured vibration by hand arm vibration promoter [6]. Electric motors and petrol/diesel engines extensively utilised for the activity of grass trimming which may cause hand arm vibration to the workers [7]. Hand arm vibration are further categorised as an industrial disease and occupies large number of workers which are associated as maintenance 
work who experiences several issues which are relevant to these vibrations [8]. Therefore, it is very much essential to develop an approach that overcome the issues caused from vibrating machines. In order to handle the vibration machines efficiently, approach is proposed that uses tuned vibration absorber.

The tuned vibration absorber, which efficiently minimises the frequent vibrations from the grass cutting machines [9]. Nodal technique is imposed which is applied to the shaft of a grass cutting machine in order to reduce the handle vibration. In order to repress the vibration in handle their technique imported to cantilevered tuned vibration absorbers. One study presents the optimisation of vibration machine design and it is noticed that the vibration is minimum by implementing analytical hierarchy process [10]. The process of analytical hierarchy is often implemented by industries for producing efficient vibrating mechanism in order to maintain the garden by providing standard among productivity and safety [11]. During the activities of gardening such as grass cutting, lifting of machine also directly affects the health of worker. The force of grass cutting machine is directly transferred to the workers hand and the engine exhaust also nearer to the nose of worker. The noise, which is generated from cutting machines, is always unpleasant and often termed as disagreeable sound [12]. Hearing loss is one of the most common disease, which is observed among the workers of garden maintenance. From one study, it is observed that out of 20 workers of garden maintenance where the noise level is in range between $85 \mathrm{dBA}$ to $93 \mathrm{dBA}$, where more than $30 \%$ of the workers were suffering from hearing loss [13]. In another study, 85 garden maintenance workers suffered from hearing loss because of the increase in noise ranges from $92 \mathrm{dBA}$ to $101 \mathrm{dBA}$, which is generated from the motorized machines [14].

The line nylon mower are the most utilized trimmers for the maintenance of garden [15]. However, from many studies it is observed that the maximum number of trimmer accident ocular were linked with these grass-cutting trimmers. The issue of ocular trauma is mainly because of improper eyewear's and other issues, which are associated within it and cannot be exaggerated. In garden trimmers having rigid blades, ocular trauma have been observed. In one study a worker is observed in ocular trauma during the switch of motor is turned off. During the cleaning process of blades, particles at the time of training gets accumulated and further causes ocular trauma. Trimming activity also may cause fugal keratitis, which is also observed from garden trimmer [16]. The nylon line garden trimmers considered as the safest among other garden maintaining trimmers because of less noise and absence of blades but studies reveals that ocular trauma may occur with such garden trimmers.

\section{Literature review}

In this section the review of noise fundamentals and its effect on health of operators are explained which is further followed by the explanation of reverse engineering, rapid development and re-engineering.

\subsection{Noise effects and its fundamentals}

The physics behind the sound and noise, defines sound as a longitudinal wave and its propagation, which generates due to the pressure vibrations near about its mean atmospheric pressure, which is generated through the vibrating surface [17]. On the other hand, noise as is termed as an unwanted and excessive noise that cause damages such as hearing loss and annoyance. Noise is a mixture of various complex pressure vibrations and there exist no easy mathematical relation among different characteristics of noise like amplitude, phase and frequency [18].

Sound is a kind of pressure fluctuation that is created from source and entirely dependent on the atmosphere at which the sources placed and its distance from source. The power of sound is the amount of energy which is transferred per unit second from the source of noise to surrounding air, and corresponds to a fixed value. The ears of human hear this sound pressure which is generated as a result of emission of sound power from sound source. Measuring instrument of sound records the pressure level from the observed sound. Because of the human perception towards mower noise which is the basis of our study, the pressure level of sound will be considered only [19].

The pressure level of sound is calculated as the logarithmic measure of rate of sound pressure from its reference value which is usually $0.00002 \mathrm{~Pa}$ where the units are in decibels. The data of noise emissions are usually provided by the manufacturers in terms of instrument's sound power along with the pressure level of sound under normal conditions [20]. Human ears can hear sound had same pressure of sound but with varying frequencies, the sound may never be heard through human ears. The measurement readings of sounds are regulated by using A-weighting [21]. The level of sound which is measured through A-weighting having units in dBA, meets the task of providing noise level measurement of single number, by integrating different sound levels of each frequency $E$ and 
providing a scale for each noise level that human ear can perceive [22].

For commercial areas the specific regulations for noise are given by the environmental management authority, which are in range of $85 \mathrm{~dB}$ during daytime and $70 \mathrm{~dB}$ during night. The limit of less safe exposure depend upon the intensity of noise, as noise intensity e is more significant lesser will be the exposure limit [23]. Table 1, presents the exposure limit for safety, which are specified by the health act where first column represents the intensity level of sounds in decibels and column to represents the safety time limit of exposure. The limit of safety time exposure at $85 \mathrm{~dB}$ is 8.5 hours, and exposure time of safety at 96 $\mathrm{dB}$ is 4.5 hours. Whenever this permitted limit exceeds it may cause damage as hearing loss, concentration loss and stress at physical and psychological level [24, 25].

Table 1: Exposure limits of safety defined by Health act

\begin{tabular}{cc}
\hline Intensity of sound in $\mathbf{d B}$ & Exposure limit per hour \\
\hline 85 & 8.5 \\
91 & 6.5 \\
96 & 4.5 \\
98 & 3.5 \\
101 & 2.5 \\
107 & 1 \\
\hline
\end{tabular}

Table 2 presents the various effect of loudness with the changing pressure level of sound. It is observed that at 4 and $6 \mathrm{~dB}$, the sound loudness is nearly, clearly observable, whereas at 12 and $25 \mathrm{~dB}$ of intensity levels, the loudness may be half or twice as loud and it may be quieter or louder [26]. Rotational and vortex are the two classifications of noise through blades. Periodic noise sometimes also referred to as rotational noise, which rises because of the distortion and effects of rotation [27]. Broadband noise sometimes also referred to as vortex noise, which arises because of the formation of vortex and turbulence. It is analysed from the experimentation that the source of most dominating noise is vortex noise through the rotating blades [28, 29]. Eq. (1) represents the passage frequency $\left(f_{b l}\right)$ of blade where $M$ indicates the amount of blades and $\Omega$ represents speed of rotation in rev/sec.

$$
f_{b l}=M \Omega
$$

The noise effect generated from blade can be reduced by sharpening the edges of blade, reducing width and thickness of blade, increasing the rotational speed of blade, or making adjustment in the curves by adding holes, winglets for breaking the air flow [30, 31].

\subsection{Integrated methodology}

The integrated methodology for garden maintenance through mowers consists of three operational design approaches, which are described below:

\subsubsection{Rapid product design}

Rapid product design consist the usage of rapid prototyping approaches for easy, fast and list costly physical design of three dimensional model. Three-dimensional model is designed by utilizing CAD (computer-aided design) software. Rapid prototyping is gaining attention and one of the growing field of development as it enables industry to facilitate effective, rapid design conversions through threedimensional models [32]. The first design of rapid prototyping is introduced in year 1987 by using lithography method. In the lithography approach, lasers were utilised in order to harden the ultraviolet light sensitive liquid layers. Later with the advancement in technology, many alternative methods are introduced like selective laser sintering, designing of laminated object and many others.

\subsubsection{Re-engineering process}

It is the process in which the product design is reconstructed in order to enhance the performance by simply modifying the material and adjusting the geometrical optimisations [33].

\subsubsection{Reverse engineering process}

It is the process in which existing object or product is modelled, evaluated and documented. Computer-aided software is utilised for the evaluation and modelling of existing products. Documented designs then tested virtually for repairing, replacing, improving or redesigning of product. In the process of reverse engineering mostly the product is handled physically where its measurement, modelling and sketching is carried out. The hand held instruments such as rulers, callipers, micrometres and skills are the tools that are utilised for measuring small range products, whereas laser scanners and optical comparators are the instrument that are utilised for measuring machines [34]. 
Table 2: Various effects of loudness with variation in pressure levels

\begin{tabular}{cc}
\hline Intensity level change & Loudness variations \\
\hline 4 & Nearly observable \\
6 & Clearly observable \\
12 & Semi or double as loud \\
25 & Far quieter or very louder \\
\hline
\end{tabular}

\section{Design methodology}

The design methodology, which is followed in this article, is depicted in Figure 1. The three different operational phases of the proposed design are reverse engineering, reengineering and rapid product design.

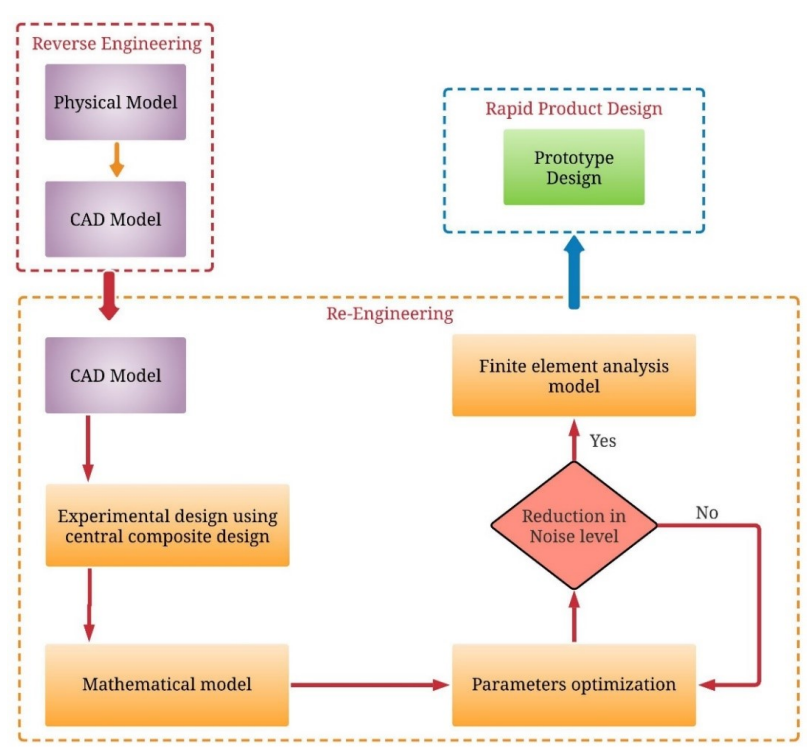

Figure 1: Integrated working flow of low noise blade design

\subsection{Phase I: Reverse engineering}

In this phase, the existing product is analysed where it is studied and then tested for making for the improvements in the key characteristics for reducing the intensity of noise. In this stage, the blade of mower is analysed for reverse engineering for making an efficient design in order to reduce the intensity of noise, which is generated from the blade. The physical specifications of mower blade are acquired by using calliper and which was further recorded by drawing photos. The dimensions of blade are utilised in order to make a three dimensional model through computer aided design software. Due to the prolonged usage of blade, some of the portion faces distortion, and complex curvature of the blade is studied by the CAD software.

\subsection{Phase II: Re-engineering}

In this phase, the virtual model is designed by the computational fluid dynamics. The rotating blade is employed in order to perform the experiments virtually considering various geometries of blade for meeting the goal of less noise intensity through blades. Various assumptions that are considered for designing the dynamics, which are described below:

\subsubsection{Assumptions:}

1) Movement of air through blade only.

2) The rotational speed of blade is 3000 RPM.

3) Angular acceleration is considered to be zero i.e., constant angular velocity.

4) The initial condition of temperature and pressure is $290 \mathrm{~K}$ and $104.2 \mathrm{kPa}$.

5) The flow of air is turbulent.

6) The dynamic pressure difference between top and bottom blade's surface is considered as sound pressure.

7) Polycarbonate is the material of blade.

During experimentation, the computational domain is reduced from its original size $0.70 \mathrm{~m}$ in $\mathrm{x}$-axis to $0.20 \mathrm{~m}$ in $\mathrm{y}$-axis and $0.82 \mathrm{~m}$ is $\mathrm{z}$-axis where the Blade is at the centre. The computational domain is reduced in order to minimise the computational time by considering less number of iterations are enough for smaller volume. After the reduction in computational domain, the rotating regions are created with the help of extruded cylinder, which consists the complete blade and represents the volume of air which rotates along with blade. The cylinder was carefully chosen as rotating area where the velocity is considered as -3000 RPM. This speed was considered based on the power of mower motor and negative sign indicates clockwise rotation. For the experimental analysis two surface goals were considered where first is the calculation of average dynamic pressure present at the top surface of blade and other is calcu- 
lation of dynamic pressure present it the bottom of blade surface.

\subsubsection{Experimental calculations:}

Calculated average pressure at top of blade surface $=$ 1102.24 Pa

Calculated average pressure at bottom of blade surface $=$ 1094.36 Pa

Pressure of sound $=1102.24 \mathrm{~Pa}-1094.36 \mathrm{~Pa}=7.88 \mathrm{~Pa}$

Level of sound pressure $=20 \log _{10}\left(\frac{P}{P_{o}}\right)=$ $20 \log _{10}\left(\frac{7.88}{0.00002}\right)=112 d B$

\subsubsection{Experimental validation:}

The noise rotational speed analysis was carried out for the verification of virtual simulation where the original blade is simulated using CAD software and then physical experiment is performed. The result obtained from both of these test are compared as depicted in Figure 2, for the determination weather the desired virtual model is adequate on the basis of vibration among observed results to predict real noise which is produced through rotating blade.

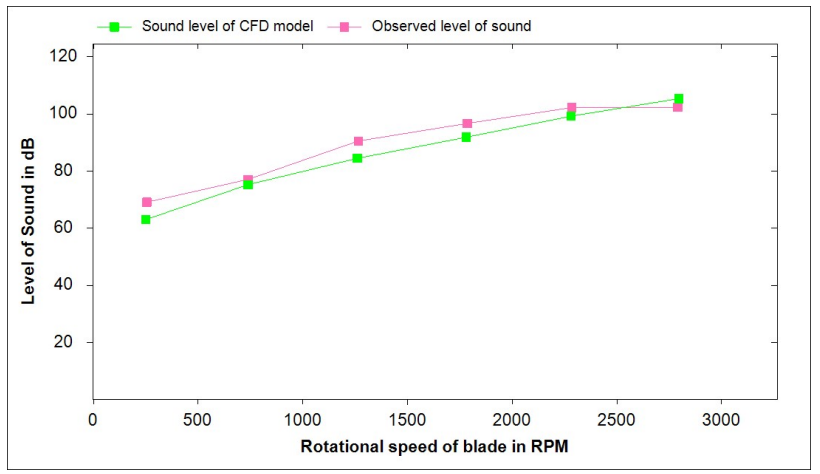

Figure 2: Sound level comparison through physical and virtual observations

Values of noise intensity levels were obtained through virtual computational fluid dynamics which where is in physical parameters ranging from $+1.4 \mathrm{~dB}$ and $5.4 \mathrm{~dB}$. From the physical experimentation which are subjected to error it is also concluded from the experimentation that virtual model are more simple in nature and because of its inapposite approximation the virtual model can be implemented for conducting subsequent experiments.

\subsection{Phase III: Rapid product design}

The third phase is rapid product design or rapid prototyping, which uses fused deposition modelling approach. The final design of the blade is sliced and printed diagonally in this final phase.

The designed blade that produces less noise and vibrations is tested and compared with the original blade for the assessment of improvement. Noise test was conducted where the first test is carried out at a very close range through to a decibel metre which was placed at decks edge and thereafter the level of noise is recorded where the mower is considered as sweeping air and cutting grass. The testing and finding results tabulated in Table 3.

The experimentation was followed by various noise distance examinations where different noise levels was measured across different distance values in range from 0 to 20 metres apart from mower cutting grass. It is observed from the experimentation that the level of noise of mower decreases with the increase in distance and the prototype blade generates less noise consistently in comparison with original blade. The difference of $5 \mathrm{~dB}$ is experienced by the operator through the prototype blade.

\section{Experimental analysis}

The gardeners, operators and maintenance staff of Municipal Corporation utilizes maintenance equipment's every day during summer. Most of the operators are unaware of potential risk that causes hearing loss and other ill effects, which may cause through the vibrations. In this study, the mowers investigated for noise intensity levels and frequency analysis of noise. The generated noise through mowers were measured using ISO 9612 where is the frequency were measured using ISO 2631 standard.

\subsection{Noise measurements}

Noise measurement outcomes at different frequency levels were utilised for the identification of specific frequency in high intensities. The identification of specific frequency level is helpful for the development of necessary control measures and selecting proper protection for ears. From the experimental analysis, it is observed that lower frequencies does not possesses any concern for industries but in case of mowers, it is a big concern. The attenuation level, which is a recommended from the industry, is in range between 11-35 $\mathrm{dB}$, which completely depend upon 
Table 3: Observed noise level at close range through both blades

\begin{tabular}{ccc}
\hline \multirow{2}{*}{ Experimental cases } & \multicolumn{2}{c}{ Observed noise in dB } \\
\cline { 2 - 3 } Computational fluid dynamics & Original blade & Designed blade \\
model & 110 & 100.12 \\
During grass cutting & 109.2 & 106.4 \\
Without grass cutting & 109 & 102.1 \\
\hline
\end{tabular}

the device of hearing protector and the content of noise frequency.

In order to reduce the level of noise various control methods and measures are utilised. When the control such as engineering and administrative does not provide any feasible solution or level of noise which is less than 80 $\mathrm{dB}$, in such cases protective devices are recommended to the workers. The hearing loss occurs because of the excessive noise, which is more than $80 \mathrm{~dB}$. For the prevention of hearing, loss it is essential to reckon noise spectral content and such device could be designed that offers personal protective measures considering the noise frequency.

\subsection{Vibration measurement}

In agriculture, vibration is one of the important health issue, which cause various hazards. $V, W$ and $A$ are the three characteristics units which are used for measuring vibrations. $V$ represents the velocity of vibration which is measured in $\mathrm{mm} / \mathrm{s}$. Eq. (2) represents the formula which is used for measuring velocity.

$$
V=2 \Pi a f
$$

Where $a$ represents the amplitude of vibration and $f$ denotes the frequency of vibration. $W$ represents the acceleration of vibration which is measured in $\mathrm{mm} \mathrm{s}^{-2}$ and it may be calculated for three axis. Eq. (3) represents the formula which is used for measuring acceleration and in this study the given acceleration in computed in RMS as mean value.

$$
W=4 \Pi^{2} a f^{2}
$$

In the experimentation, we have considered daily 7 hours of gardening. The calculation exposure level of vibration which is transferred to the hand-arm is determined on the basis of everyday exposure i.e., 7 hours daily as reference period. Eq. (4) represent the exposure time of hand-arm vibrations.

$$
A(7)=A_{(W) \operatorname{sum}}\left(\frac{T e}{7}\right)^{\frac{1}{2}}
$$

Equation 4, $\left(\mathrm{m} A(7) \mathrm{s}^{-2}\right)$ is evaluated on the basis of root of sum of square $A_{(W) \text { sum }}$ of the value of root mean square of frequency accelerations which is calculated on three different axis $\mathrm{x}, \mathrm{y}$ and $\mathrm{z}$. The equation to evaluate is $A(7)$ presented in Eq. (4), where Te represents the exposure of everyday vibration in hours.

$$
A_{(W) \text { sum }}=\left(a_{W X}^{2}+a_{W Y}^{2}+a_{W Z}^{2}\right)^{\frac{1}{2}}
$$

Eq. (5) represent the calculation of RMS value of frequency acceleration. $a_{W X}, a_{W Y}$ and $a_{W Z}$ represents the sum of RMS values on $\mathrm{x}, \mathrm{y}$ and $\mathrm{z}$-axis.

\section{Results and analysis}

It is observed from the experimentation that the regular vibration exposure cause some serious health issues. The experimental results signifies the difference among the noise and vibration values generated through vehicle, lawn mowers and mowers of riding capacity. The safest among all of these system is mower with riding capacity where observed noise level is under $1.2 \mathrm{~m}\left(\mathrm{~s}^{2}\right)^{-1}$. On the other hand, lawn mowers and vehicles gave higher levels of vibration which is observed over $2 \mathrm{~m}\left(\mathrm{~s}^{2}\right)^{-1}$. The observed noise and frequency from vehicle users also exceed the limit of regulating, which limits their use in public. More specifically the vehicles are used for towing heavy loads. The equipment for personal protection has to be used by all operators for such heavy machines.

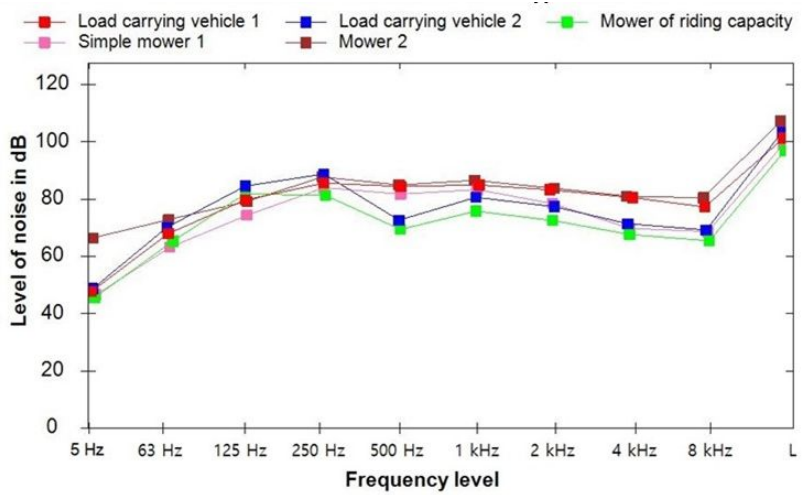

Figure 3: Vibration noise content due to various types of emissions 
Table 4: Observed values of vibration through various machines

\begin{tabular}{cccc}
\hline Type of machine & \multicolumn{3}{c}{ Vibration analysis } \\
\cline { 2 - 4 } & $\begin{array}{c}\text { Amplitude (A) } \\
\text { in } \mathrm{mm}\end{array}$ & $\begin{array}{c}\text { Velocity (V) in } \\
\mathrm{mm} \mathrm{s}^{-1}\end{array}$ & $\begin{array}{c}\text { Acceleration (W) } \\
\text { in } \mathrm{m}^{\left(\mathrm{s}^{2}\right)^{-1}}\end{array}$ \\
\hline Load carrying vehicle 1 & 0.05 & 6.9 & 0.97 \\
Load carrying vehicle 2 & 0.081 & 9.1 & 1.02 \\
Mower with riding capacity & 0.052 & 2.5 & 0.15 \\
Simple mower 1 & 0.132 & 23.1 & 3.68 \\
Mower 2 & 0.119 & 19.02 & 2.66 \\
\hline
\end{tabular}

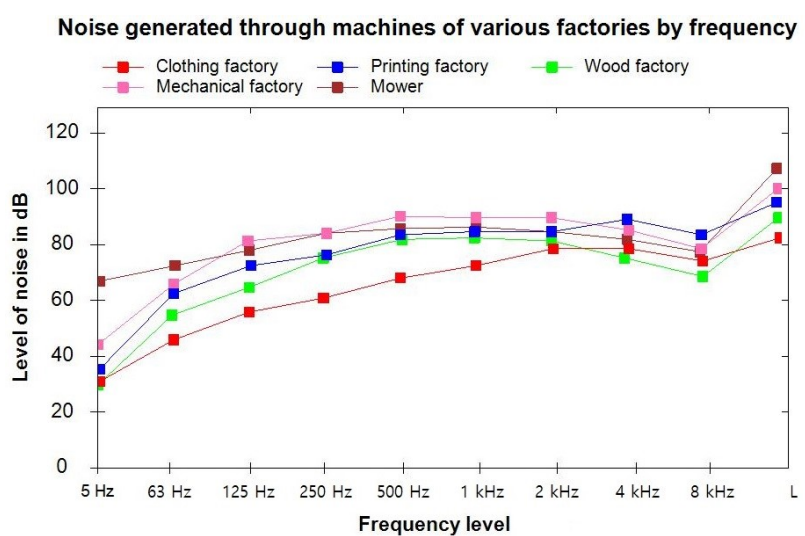

Figure 4: Level of noise from machines of various factories at different frequency levels

Vibrational noise content from various type of emissions is depicted in Figure 3. The experimental analysis enables operator to select right kind of earmuffs through noise frequency for the prevention from hearing loss. Experiments also extended in order to make the comparison for different noise level through gardening and industrial machines. The comparison of various noise through various factories and industrial instruments is depicted in Figure 4. The measurement of noise from manufacturing industries for carried out from different case studies, which are as follows: textile industry, wood industry, printing and mechanical industry. Noise is observed as the most common health hazard in four various industries (printing, mechanical, textile and wood). The different noise spectrum is also observed during the vibration and noise analysis of these industries. The level of noise from clothing industry is observed as lower in comparison with other four industries. On comparing these outcomes with noise generated from machines of lawn maintenance, it is predictable that at lower frequencies in range between $32 \mathrm{~Hz}$ to $64 \mathrm{~Hz}$ the level of noise is higher in range between 18 to $30 \mathrm{~dB}$. Therefore, they are more dangerous than the machines are used for manufacturing. Table 4, presents the observe vibration on seat of vehicles and mowers of riding capacity through the hand grip.

\section{Conclusion}

This article presented an integrated approach for designing testing and developing mower blade that generates less noise. The differences in the values of noise and vibration are observed through obtained results for load carrying vehicles, mowers with riding capacity and simple mowers. From the analysis, mower with riding capacity is observed as safest among all other types of machines as it provides the value of vibration below $1.2 \mathrm{~m}\left(\mathrm{~s}^{2}\right)^{-1}$. The higher level of vibrations are obtained from simple mowers which is over $3.1 \mathrm{~m}\left(\mathrm{~s}^{2}\right)^{-1}$. The measurement of noise from manufacturing industries for carried out from different case studies, which are as follows: textile industry, wood industry, printing and mechanical industry. Noise is observed as the most common health hazard in four various industries (printing, mechanical, textile and wood). By implementing this proposed design, the operational value of noise for a battery-powered mower reduces by $7.88 \mathrm{~dB}$ then measure for system without grass cutting, $2.8 \mathrm{~dB}$ when measured at deck's edge and $4.7 \mathrm{~dB}$ when measured during grass cutting.

Funding information: The authors state no funding involved.

Author contributions: All authors have accepted responsibility for the entire content of this manuscript and approved its submission.

Conflict of interest: The authors state no conflict of interest.

\section{References}

[1] McCallig M, Paddan G, Van Lente E, Moore K, Coggins M. Evaluating worker vibration exposures using self-reported and direct observation estimates of exposure duration. Appl Ergon. 2010;42(1):37-45.

[2] Coggins MA, Van Lente E, McCallig M, Paddan G, Moore 
K. Evaluation of hand-arm and whole-body vibrations in construction and property management. Ann Occup Hyg. 2010;54(8):904-14.

[3] Atkins T. The science and engineering of cutting: the mechanics and processes of separating, scratching and puncturing biomaterials, metals and non-metals. ButterworthHeinemann; 2009.

[4] Armstrong D. A survey of community gardens in upstate New York: implications for health promotion and community development. Health Place. 2000;6(4):319-27.

[5] Morgenroth J, Santos B, Cadwallader B. Conflicts between landscape trees and lawn maintenance equipment-The first look at an urban epidemic. Urban For Urban Green. 2015;14(4):1054-8.

[6] Lee HY, Tseng HH, Zheng MC, Li PY. Decision support for the maintenance management of green areas. Expert Syst Appl. 2010;37(6):4479-87.

[7] Kean AJ, Sawyer RF, Harley RA. A fuel-based assessment of off-road diesel engine emissions. J Air Waste Manag Assoc. 2000;50(11):1929-39.

[8] Ayers B, Forshaw M. An interpretative phenomenological analysis of the psychological ramifications of hand-arm vibration syndrome. J Health Psychol. 2010;15(4):533-42.

[9] Azmir NA, Ghazali MI, Yahya MN, Ali MH. Hand-arm vibration disorder among grass-cutter workers in Malaysia. Int J Occup Saf Ergon. 2016;22(3):433-8.

[10] Azmir NA, Ghazali MI, Yahya MN, Ali MH, Song JI. Effect of hand arm vibration on the development of vibration induce disorder among grass cutter workers. Procedia Manuf. 2015;2:87-91.

[11] Tilvikiene V, Kadziuliene Z, Dabkevicius Z, Venslauskas K, Navickas K. Feasibility of tall fescue, cocksfoot and reed canary grass for anaerobic digestion: analysis of productivity and energy potential. Ind Crops Prod. 2016;84:87-96.

[12] Mallick Z, Badruddin IA, Khaleed Hussain MT, Salman Ahmed $\mathrm{NJ}$, Kanesan J. Noise characteristics of grass-trimming machine engines and their effect on operators. Noise Health. 2009;11(43):98-102.

[13] Tint P, Tarmas G, Koppel T, Reinhold K, Kalle S. Vibration and noise caused by lawn maintenance machines in association with risk to health. Agron Res. 2012;10(1):251-60.

[14] Azmir NA, Ghazali MI, Yahya MN, Ali MH. Hand-arm vibration disorder among grass-cutter workers in Malaysia. Int J Occup Saf Ergon. 2016;22(3):433-8.

[15] Yaovaja K, Bamrungthai P, Ketsarapong P. Design of an autonomous tracked mower robot using vision-based remote control. 2019 IEEE Eurasia Conference on IOT, Communication and Engineering (ECICE). 2019 Oct 3-6; Yunlin, Taiwan. IEEE. 2019; p. 324-327.

[16] Kamaruzzaman A, Ibrahim TA, Sulaiman FR, Ismail I. Occupational noise exposure and the effects on blood pressure of grass-cutting workers. IOP Conf Ser:: Earth Environ Sci. 2021;685(1):012002.

[17] Ulhe PP, Inwate MD, Wankhede FD, Dhakte KS. Modification of solar grass cutting machine. Int J Innovative Res Sci Technol 2016;2(11).

[18] Fahmi R, Bridgwater AV, Thain SC, Donnison IS, Morris PM, Yates N. Prediction of Klason lignin and lignin thermal degradation products by Py-GC/MS in a collection of Lolium and Festuca grasses. J Anal Appl Pyrolysis. 2007;80(1):16-23.
[19] Walker BN, Lindsay J. Navigation performance with a virtual auditory display: effects of beacon sound, capture radius, and practice. Hum Factors. 2006;48(2):265-78.

[20] Shah AD, Mujawar SJ, Sutar PR, Prasad SR. Solar Powered Intelligent Grass Cutter Robot. International Journal of Scientific Development and Research. 2020;5:229-34.

[21] Zastrow C. Understanding and preventing burn-out. Br J Soc Work. 1984;14(1):141-55.

[22] Kumar A, Mathur NN, Varghese M, Mohan D, Singh JK, Mahajan P. Effect of tractor driving on hearing loss in farmers in India. Am J Ind Med. 2005;47(4):341-8.

[23] Levey S, Fligor BJ, Ginocchi C, Kagimbi L. The effects of noiseinduced hearing loss on children and young adults. Contemp Issues Commun Sci Disord. 2012;39(Fall):76-83.

[24] Jaafar NI, Daud MK, Mohammad I, Abd Rahman N. Noiseinduced hearing loss in grass-trimming workers. Egyptian Journal of Ear, Nose. Throat and Allied Sciences. 2017;18(3):227-9.

[25] Sekhon NK, Masterson EA, Themann CL. Prevalence of hearing loss among noise-exposed workers within the services sector, 2006-2015. Int J Audiol. 2020;59(12):948-61.

[26] Balanay JA, Kearney GD, Mannarino AJ. Noise exposure assessment among groundskeepers in a university setting: $A$ pilot study. J Occup Environ Hyg. 2016;13(3):193-202.

[27] Dewangan KN, Kumar GP, Tewari VK. Noise characteristics of tractors and health effect on farmers. Appl Acoust. 2005 Sep;66(9):1049-62.

[28] Jaafar NI. Noise Induced Hearing Loss Among Grass Trimming [dissertation], Universiti Sains Malaysia.

[29] Walker BN, Lindsay J. Navigation performance with a virtual auditory display: effects of beacon sound, capture radius, and practice. Hum Factors. 2006;48(2):265-78.

[30] Roll P, Klintschar M. Fatal missile injury from the rotating knife of an agricultural mower. Forensic Sci Int. 1998;94(1-2):1-8.

[31] Yang J, Sharma A, Kumar R. IoT-Based Framework for Smart Agriculture [IJAEIS]. Int J Agric Environ Inf Syst. 2021;12(2):114.

[32] Ko YH, Ean OL, Ripin ZM. The design and development of suspended handles for reducing hand-arm vibration in petrol driven grass trimmer. Int J Ind Ergon. 2011;41(5):459-70.

[33] Yan Y, Zhang Y, Sharma A, Al-Amri JF. Evaluation of Suitability of Urban Land Using GIS Technology. Sustainability (Basel). 2021;13(19):10521.

[34] Kulhariya SK, Tomer AS, Krishan K, Yadav NK, Mishra RK. Performance evaluation of solar operated bidirectional grass cutter. J Pharmacogn Phytochem. 2020;9(3):329-34. 